\title{
EDITORIAL
}

\section{The interventricular septum is functionally bilayered: a fresh look at a well known structure}

\section{F A Flachskampf, J-U Voigt}

Heart 2005;91:1260-1261. doi: 10.1136/hrt.2004.057646

\section{Increasingly sophisticated echocardiographic procedures are revealing previously unknown details of well known structures such as the interventricular septum}

$\mathrm{T}$ he interventricular septum* is among the cardiac structures best visualised by echocardiography, even in poorly echogenic patients. In the four chamber view, but also in some patients in the parasternal view, a highly echogenic, thin central zone is seen in the septum, coursing from base to apex. Oddly enough, this zone or line has not received much attention in the past.

In this issue of Heart, Boettler et al ${ }^{1}$ hypothesise that this line separates right ventricular and left ventricular components of the septum, and that these two components or layers are functionally different, as demonstrable by tissue Doppler methods. The increased echogenicity of the separating line may be due to an abrupt change in myocardial fibre orientation, septal coronary vessels, or both. To test this hypothesis, they analysed echocardiographic recordings from 30 healthy individuals without septal hypertrophy and supplemented their findings with three examples of "unilateral" septal pathology.

Their findings can be summarised as follows:

- a highly echogenic central septal zone was found in all individuals, coursing from the apex of the left ventricle to the aortic root

- left ventricular and right ventricular components of the septum are different: the left ventricular myocardium is thicker $(6 \mathrm{~mm} v$ $4 \mathrm{~mm}$ at end diastole at the mid septal level) and thickens more (49\% $v$ 17\%)

- tissue Doppler revealed higher radial strain (that is, thickening; $41 \% \vee 22 \%$ ) and strain rate $(1.1 / \mathrm{s} \vee 0.5 / \mathrm{s})$ on the left septal side; in contrast, longitudinal strain (base-to-apex shortening) and strain rates differed only minimally.

See end of article for authors' affiliations

Correspondence to: Frank A Flachskampf, MD, Med. Klinik II, Universitätsklinikum Erlangen, Ulmenweg 18, 91054 Erlangen, Germany; frank. flachskampf@rzmail. uni-erlangen.de

\section{CLINICAL IMPORTANCE}

Why is this important clinically? We use to think of the interventricular septum as one structure. For example, in every complete echocardiographic examination end diastolic "total" septal thickness is measured as an indicator of left ventricular mass. In the light of the present findings, this practice is an oversimplification, because left ventricular hypertrophy should primarily affect the left ventricular component of the septum. On the other hand, the right ventricular component of the septum, which is much better visualised by echo than the right ventricular free wall, may contain important information on right ventricular function and disease. Detection of right ventricular ischaemia and infarction may be improved by scrutinising the right ventricular portion of the septum. Similar to the well demonstrated value of tissue Doppler for the early detection of left ventricular myocardial diseases such as diabetic cardiomyopathy, ${ }^{2}$ amyloidosis, ${ }^{3}$ or Fabry disease, ${ }^{4}$ deformation imaging (that is, strain/strain rate imaging) by tissue Doppler may be valuable for detecting right ventricular disorders such as arrhythmogenic right ventricular dysplasia, myocardial effects of pulmonary valve disease, ventricular and atrial septal defect, etc. Animal experiments have shown that right ventricular strain responds to changes in right ventricular loading conditions and that there is regional variation in right ventricular myocardial function. ${ }^{5}$

Of course, there are limitations to this approach. Although the authors make a convincing case for functional heterogeneity of the interventricular septum, the physical continuity of the two components attached to each other by a large interface makes strong interaction likely. This certainly contributes to the similarity of longitudinal strain and strain rate of the two septal components.

\section{WALL STRESS}

Another factor affecting regional septal function is wall stress. Given the geometry and pressure levels of the two ventricles, by LaPlace's law systolic wall stress increases with radius, and thus the right ventricular side of the septum will experience a higher systolic wall stress (higher afterload) than the left ventricular side. This may in part explain functional differences. It should also be remembered that the thickness of each of these components is in the order of magnitude of the spatial resolution of deformation imaging, which is based on the difference of tissue velocities a few millimetres apart. Cardiac motion further compounds the problem of accurately positioning a sample volume exclusively in one part of the 
septum. It should also be kept in mind that the morphologic substrate of the bright mid septal zone remains speculative, as well as whether this is precisely the border of right ventricular and left ventricular septal components.

Nevertheless, the present work shows that the ongoing sophistication of echocardiographic methods brings out relevant, but heretofore inaccessible information from seemingly well explored and understood cardiac structures.

\section{Authors' affiliations}

F A Flachskampf, J-U Voigt, Med. Klinik II, Universitätsklinikum Erlangen, Erlangen, Germany

\section{REFERENCES}

Boettler $\mathbf{P}$, Claus $\mathrm{P}$, Herbots $\mathrm{L}$, et al. New aspects of the ventricular septum and its function: an echocardiographic study. Heart 2005;91:1343-8.

2 Fang ZY, Najos-Valencia O, Leano R, et al. Patients with early diabetic heart disease demonstrate a normal myocardial response to dobutamine. J Am Coll Cardiol 2003;42:446-53.

3 Koyama J, Ray-Sequin PA, Falk RH. Longitudinal myocardial function assessed by tissue velocity, strain, and strain rate tissue Doppler echocardiography in patients with AL (primary) cardiac amyloidosis. Circulation 2003; 107:2446-52

4 Weidemann F, Breunig F, Beer $M$, et al. Improvement of cardiac function during enzyme replacement therapy in patients with Fabry disease: a prospective strain rate imaging study. Circulation 2003;108:1299-301

5 Jamal $F$, Bergerot C, Argaud L, et al. Longitudinal strain quantitates regional right ventricular contractile function. Am J Physio 2003;255:H2842-7.

\section{IMAGES IN CARDIOLOGY}

\section{Haemodynamic monitoring with a left ventricular assist device}

A

50 year old woman with a history of end stage non-ischaemic cardiomyopathy presents with two days of increasing dyspnoea at rest and orthopnoea. The patient was hypoxic, hypotensive, and tachycardic. Clinically, the patient was felt to have decompensated heart failure so she was admitted to the cardiac intensive care unit for haemodynamic monitoring and management.

Invasive haemodynamic monitoring and intensive medical treatments were initiated. Despite maximal medical treatment the patient did not improve, so a more invasive strategy to provide haemodynamic support was pursued. The figures here demonstrate haemodynamic tracings (below) and a schematic representation (right) of one such invasive device used for haemodynamic support.
The tracings below depict simultaneous ECG, radial arterial pressure tracing, pulmonary artery pressure tracing, and central venous pressure tracing being monitored in the cardiovascular intensive care unit. The tracings show total dissociation between the radial arterial pressure tracing and the heart rhythm as denoted by the ECG. While the radial arterial waveform shows no relation to the ECG, the right heart pressures as recorded by the pulmonary artery pressure and the central venous pressure are synchronous with the ECG. The reason for the dysynchrony between the radial arterial pressure and the intrinsic cardiac cycle is a mechanical left ventricular assist device (LVAD) has been implanted. The LVAD in demand mode cycles when it fills, with timing that is independent of the native cardiac cycle. Thus the radial

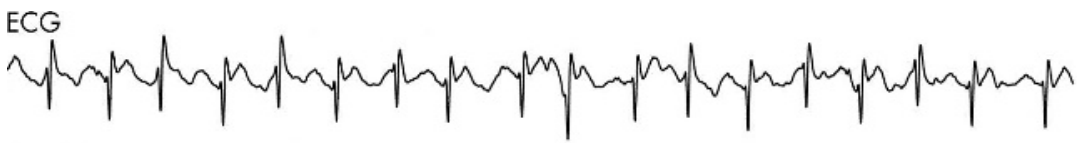

Arterial pressure

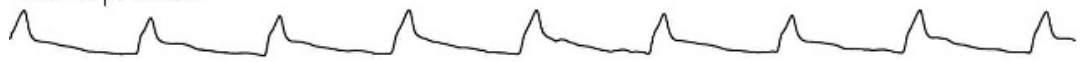

Pulmonary artery pressure

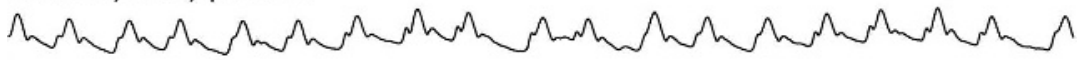

Central venous pressure

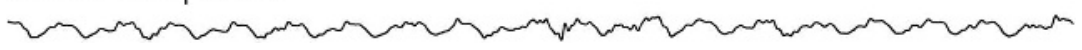

doi: $10.1136 /$ hrt.2004.057786

arterial pressure waveforms have timing that is independent of the native right sided circulation and the intrinsic cardiac rhythm.

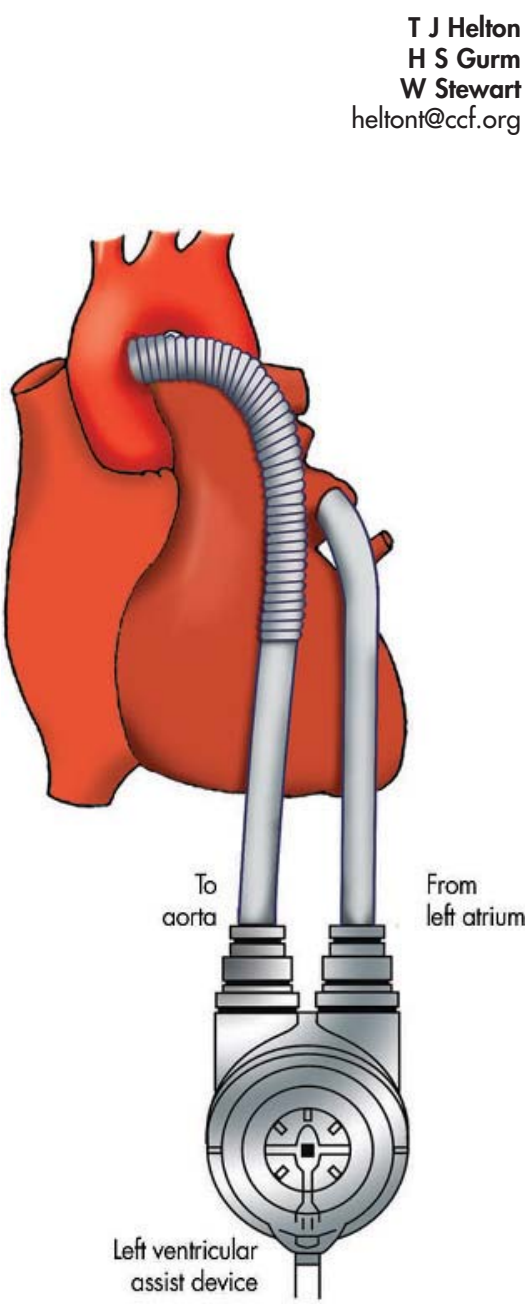

